\title{
Correction to: A Bayesian Model for Estimating the Effects of Human Disturbance on Wildlife Habitats Based on Nighttime Light Data and INLA-SPDE
}

\author{
Changbai Xi ${ }^{1}$. Zhaoning Wu ${ }^{1}$. Tianlu Qian ${ }^{1} \cdot$ Lu Liu $^{1}$. Jiechen Wang ${ }^{1,2}(\mathbb{C}$ \\ Published online: 16 February 2022 \\ (c) Springer Nature B.V. 2022
}

\section{Correction to: Applied Spatial Analysis and Policy https://doi.org/10.1007/s12061-021-09402-6}

In the original version the authors unintentionally omitted reference to the work of Humphreys et.al (2020). This is corrected at the end of paragraph 6 in the Introduction:

"These advantages have made it successful in ecological, soil, and epidemiological studies (Huang et al., 2017; Innocent et al., 2017; Poggio et al., 2016). Further, Humphreys et al. (2020) developed a novel geostatistical method, taking advantage of Gaussian random fields, to estimate human disturbance in wetlands and coastal waters."

The citation of the article is:

"Humphreys, J. M., Mahjoor, A., Reiss, K. C., Uribe, A. A., \&Brown, M. T. (2020). A Geostatistical Model for Estimating Edge Effects and Cumulative Human Disturbance in Wetlands and Coastal Waters. International Journal of Geographical Information Science, 34(8), 1508-1529. https://doi.org/10.1080/13658816.2019.1577431."

The authors regret this error.

The original article has been corrected.

Publisher's Note Springer Nature remains neutral with regard to jurisdictional claims in published maps and institutional affiliations.

The original article can be found online at https://doi.org/10.1007/s12061-021-09402-6.

Jiechen Wang

wangjiechen@nju.edu.cn; jiechen_wang@outlook.com

Changbai Xi

xicb11@smail.nju.edu.cn 
Zhaoning Wu

wzngeo@smail.nju.edu.cn

Tianlu Qian

tianluqian@outlook.com

Lu Liu

liulu@smail.nju.edu.cn

1 Jiangsu Provincial Key Laboratory of Geographic Information Science and Technology, Key Laboratory for Land Satellite Remote Sensing Applications of Ministry of Natural Resources, School of Geography and Ocean Science, Nanjing University, 163 Xianlin Road, Qixia District, Nanjing 210023, Jiangsu, China

2 Jiangsu Center for Collaborative Innovation in Geographical Information Resource Development and Application, Nanjing 210023, China 